may be to use this as a covariate in casecontrol studies and examine whether risk is modified. This would require that cases and controls had appropriate genetic material available for analysis and would probably require a prospective study.

\section{Mark Weatherall}

Correspondence to Dr Mark Weatherall, Wellington School of Medicine and Health Sciences, Department of Medicine, PB 7343, Wellington South, Wellington 6242, New Zealand; mark.weatherall@otago.ac.nz

Competing interests None.

Provenance and peer review Not commissioned; not externally peer reviewed.

Accepted 24 August 2010

Published Online First 14 October 2010

Thorax 2011;66:542-543.

doi:10.1136/thx.2010.149765

\section{REFERENCES}

1. Weatherall M, Wijesinghe $\mathbf{M}$, Perrin $\mathrm{K}$, et al. Meta-analysis of the risk of mortality with salmeterol and the effect of concomitant inhaled corticosteroid therapy. Thorax 2010;65:39-43.

2. Williamson PA, Short PM, McKinlay L, et al. $\beta$-agonist safety and the elephant in the room? Thorax 2011;66:542.

Transbronchial needle aspiration in the diagnosis of mediastinal amyloidosis

We read with interest the pulmonary puzzle by Khor et $a l^{1}$ on mediastinal lymph node amyloidosis diagnosed by direct real-time endobronchial ultrasound-guided (EBUS) transbronchial needle aspiration (TBNA). The development of EBUS-TBNA is improving the diagnostic yield of mediastinal lymphadenopathy. ${ }^{2}$ However, we offer some comments about the diagnostic approach used in the case described.

First, in our opinion, conventional TBNA continues to have a significant value in diagnosing mediastinal node involvement, especially in cases of lymph nodes with a short axis $>1 \mathrm{~cm}$ that are readily accessible. ${ }^{3}$ The potential advantages of TBNA over EBUS-TBNA are the lower cost, routine availability, ease of mastery, reduced need for patient sedation and the possibility of using histology needles that allow acquisition of a core of tissue, improving diagnostic sensitivity for other lesions such as tuberculosis, lymphoma and sarcoidosis. ${ }^{3}$ Our group reported the first case of mediastinal amyloidosis diagnosed by TBNA using a histology TBNA needle. ${ }^{4}$ In our opinion, according to the characteristics of the patient described and CT chest images, the safest and most cost-effective probe that was indicated in this case was TBNA using a histology needle. Second, there is a previous report in the literature of mediastinal amyloidosis diagnosis made with EBUS-TBNA ${ }^{5}$ so the report was not, as the authors stated, the first published case. Finally, we agree with the authors that, although mediastinoscopy is still considered the 'gold standard' diagnostic approach for mediastinal nodal amyloidosis, conventional TBNA sampling has value and should be considered as a less costly alternative that is universal and accessible to any bronchoscopist compared with EBUS-TBNA. In fact, it should be considered the first step in the diagnostic sequence. The debate over the role of conventional TBNA in the era of EBUS remains unresolved.

\section{Virginia Leiro-Fernández, Maribel Botana-Rial Cristina Represas, Alberto Fernández-Villar}

Investigation Group in Respiratory and Infectious diseases, Pulmonary Department, Complejo Hospitalario Universitario Vigo (CHUVI), Instituto de investigación Biomédica Vigo (IBIV), Spain

Correspondence to Virginia Leiro-Fernández, Pulmonary Department, Complejo Hospitalario Universitario de Vigo c/ Pizarro, n 22. 36204 Vigo, Pontevedra, Spain; virginia.leiro.fernandez@sergas.es

\section{Competing interests None.}

Patient consent Obtained.

Provenance and peer review Not commissioned; not externally peer reviewed.

Accepted 6 May 2010

Published Online First 17 August 2010

Thorax 2011;66:543. doi:10.1136/thx.2010.138628

\section{REFERENCES}

1. Khor YH, Steinfort DP, Buchanan MR, et al A 69-year-old smoker with mediastinal and hiliar lymphadenopathy. Thorax 2010;65:132-8.

2. Varela-Lema L, Fernández-Villar A, Ruano-Ravina A. Effectiveness and safety of endobronchial ultrasound-transbronchial needle aspiration: a systematic review. Eur Respir J 2009;33:1156-64.

3. Patel NM, Pohlman A, Husain A, et al. Conventional transbronchial needle aspiration decreases the rate of surgical sampling of intrathoracic lymphadenopathy. Chest 2007:131:773-8.

4. Leiro V, Fernández-Villar A, Bandrés R, et al. Primary amyloidosis involving mediastinal lymph nodes: diagnosis by transbronchial needle aspiration. Respiration 2008;76:218-20.

5. Fujiwara T, Yasufuku K, Nakajima T, et al. Primary amyloidosis involving mediastinal lymph nodes diagnosed by EBUS-TBNA. Respir Med 2009;2:51-3.

Implementing the change in National Institute for Health and Clinical Excellence guidance on aifflow obstruction grading in chronic obstructive pulmonary disease

The updated National Institute for Health and Clinical Excellence (NICE) chronic obstructive pulmonary disease (COPD) guidelines ${ }^{1}$ and the draft national strategy for $\mathrm{COPD}^{2}$ have recommended a change in the classification of airflow obstruction severity to align them with international classifications. NICE's 2004 guidelines recognised that disease severity is not the same as the severity of airflow obstruction and has recommended using other measures such as the Medical Research Council (MRC) dyspnoea scale, exacerbation frequency and multicomponent indices. ${ }^{3}$ However, UK primary care has been encouraged to code disease severity into mild, moderate and severe COPD based on lung function alone in line with NICE's 2004 guidance on airflow obstruction.

The code for COPD is thus H3; H36 is mild COPD; H37 is moderate COPD and $\mathrm{H} 38$ is severe COPD.

A person with COPD and an forced expiratory volume in $1 \mathrm{~s}$ of $42 \%$ of predicted has until now been coded as having moderate COPD; according to NICE 2010 they should now be coded as severe airflow obstruction. However, codes do not exist for mild, moderate, severe and very severe airflow obstruction. Therefore, for both patients and primary clinicians we have a communication problem and a coding problem. Clear guidance is needed on how the disease/airflow obstruction severity should be coded on primary care records without any conflicting or confusing advice.

Perhaps the answer is to abolish the codes for mild moderate and severe COPD and for new codes for airflow obstruction based on GOLD stages $1-4$ to be generated. For practical purposes of classifying COPD severity, for example, for deciding the frequency of reviews, the MRC dyspnoea scale could replace H36-8 as markers of disease severity. The MRC scale is already being recorded in primary care. In future, COPD severity codes should be based on multicomponent indices, at present a suitable index for primary care has not been chosen. The NICE guidelines recommend the use of the BODE index when its component items are available, the need for the six minute walking test will make this impractical for routine use in primary care and there is insufficient evidence to approve newer indices such as the $\mathrm{ADO}^{4}$ and DOSE. ${ }^{5}$

Action is required now to address both the coding and communication issues so that the sensible advice from NICE can be implemented without causing confusion in primary care and distress to patients.

\section{Rupert C M Jones, ${ }^{1}$ Kevin Gruffydd-Jones, ${ }^{2}$ David B Price ${ }^{3}$}

'Peninsula Medical School, Respiratory Research Unit, Plymouth, UK; ${ }^{2}$ Box Surgery, London Road, Corsham, UK: ${ }^{3}$ University of Aberdeen, Department of General Practice and Primary Care, Aberdeen, UK

Correspondence to Dr Rupert C M Jones, Peninsula Medical School, Room N21, 1 Davy Road, Plymouth PL6 8BX, UK; rupert.jones@pms.ac.uk

Competing interests None to declare. 
Provenance and peer review Not commissioned; not externally peer reviewed.

Accepted 5 August 2010

Published Online First 14 October 2010

Thorax 2011:66:543-544.

doi:10.1136/thx.2010.148510

\section{REFERENCES}

1. National Clinical Guideline Centre. Chronic obstructive pulmonary disease: management of adults with chronic obstructive pulmonary disease in primary and secondary care. Updated. London: National Clinical Guideline Centre, 2010

2. Department of Health/Respiratory Team.

Consultation on a strategy for services for chronic obstructive pulmonary disease (COPD) in England. London, Department of Health, 2010.

3. National Collaborating Centre for Chronic Conditions. Chronic obstructive pulmonary disease. National clinical guideline on management of chronic obstructive pulmonary disease in adults in primary and secondary care. Thorax 2004;59 (Suppl 1):1-232.

4. Puhan MA, Garcia-Aymerich J, Frey M, et al. Expansion of the prognostic assessment of patients with chronic obstructive pulmonary disease: the updated BODE index and the ADO index. Lancet 2009;374:704-11.

5. Jones RC, Donaldson GC, Chavannes NH, et al. Derivation and validation of a composite index of severity in chronic obstructive pulmonary disease: the DOSE index. Am J Respir Crit Care Med 2009;180:1189-95.

Reversed halo sign in pulmonary zygomycosis

We read with interest the article 'The atoll sign' by Walsh and Roberton ${ }^{1}$ in the November issue of Thorax. The authors report a case of cryptogenic organising pneumonia with the atoll sign, also called the reversed halo sign. As mentioned by the authors, this CT sign was first described in cryptogenic organising pneumonia and was initially considered to be specific for this disease. ${ }^{2}$ It was subsequently reported in a variety of pulmonary diseases, including paracoccidioidomycosis (South American blastomycosis), tuberculosis, pulmonary sarcoidosis, lymphomatoid granulomatosis, Wegener's granulomatosis, lipoid pneumonia and pneumococcal pneumonia. ${ }^{3-5}$

We would like to highlight another important cause of the reversed halo sign: invasive pulmonary fungal infections, particularly pulmonary zygomycosis (PZ) the presence of the reversed halo sign on CT should be considered as invasive fungal disease until proven otherwise. It is an early sign that is more frequently seen in patients with PZ than invasive pulmonary aspergillosis (IPA). ${ }^{6}$

Early institution of high-dose antifungal therapy is associated with improved outcomes; therefore, early recognition of invasive fungal disease is important. Moreover, because the therapy for presumed fungal pneumonia in this population is often aimed at IPA due to its higher incidence and the preferred antifungal agent for IPA is voriconazole, which is not effective against $\mathrm{PZ}$, it is important to differentiate between the two entities. The presence of the reversed halo sign can be used to optimise antifungal therapy to cover PZ.

\section{Myrna C B Godoy, Edith M Marom}

University of Texas M.D. Anderson Cancer Center, Department of Diagnostic Radiology, Houston, Texas, USA

Correspondence to Myrna C B Godoy, University of Texas M.D. Anderson Cancer Center, Department of Diagnostic Radiology, 1515 Holcombe Blvd., Unit 371 Houston, TX 77030, USA; mgodoy@mdanderson.org (figure 1). In immunosuppressed patients,

\section{Competing interests None.}

Provenance and peer review Not commissioned; not externally peer reviewed.

Accepted 26 January 2011

Published Online First 8 March 2011

Thorax 2011;66:544. doi:10.1136/thx.2010.157776

\section{REFERENCES}

1. Walsh SL, Roberton BJ. Images in thorax. The atoll sign. Thorax 2010;65:1029-30.

2. Voloudaki AE, Froudarakis ME, Datseris GE, et al. Crescentic and ring-shaped opacities. CT features in two cases of bronchiolitis obliterans organizing pneumonia (BOOP). Acta Radiol

1996; 37:889-92.

3. Gasparetto EL, Escuissato DL, Davaus T, et al. Reversed halo sign in pulmonary paracoccidioidomycosis. AJR Am J Roentgenol 2005:184:1932-4.

4. Marchiori E, Grando RD, Simoes Dos Santos CE, et al Pulmonary tuberculosis associated with the reversed halo sign on high-resolution CT. Br J Radiol 2010;83:e58-60.

5. Tzilas V, Provata A, Koti A, et al. The "reversed halo" sign in pneumonococcal pneumonia: a review with a case report. Eur Rev Med Pharmacol Sci 2010;14:481-6.

6. Wahba H, Truong MT, Lei X, et al. Reversed halo sign in invasive pulmonary fungal infections. Clin Infect Dis 2008;46:1733-7

\section{Serum 25-hydroxy vitamin D and exercise capacity in COPD}

Janssens and colleagues $^{1}$ have recently reported that vitamin $\mathrm{D}$ deficiency is very common in patients affected by chronic obstructive pulmonary disease (COPD) and that vitamin $\mathrm{D}$ status correlates with lung function. In the same issue of Thorax, Quint and Wedzicha ${ }^{2}$ discuss potential effects of vitamin $\mathrm{D}$ deficiency and supplementation

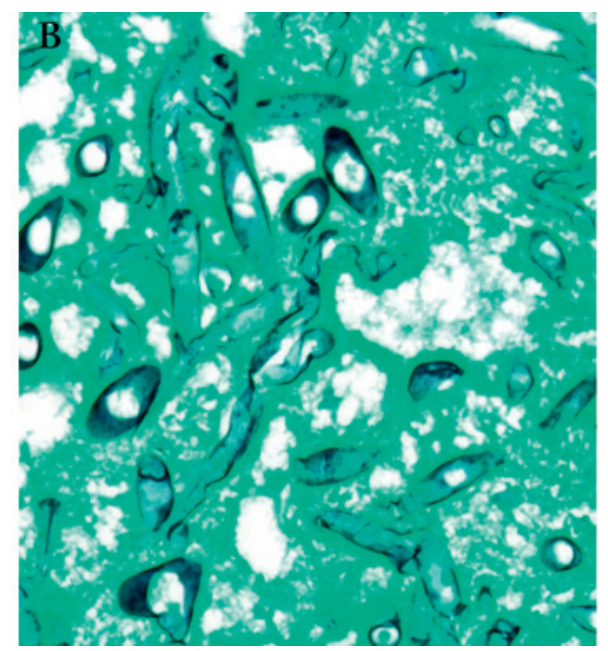

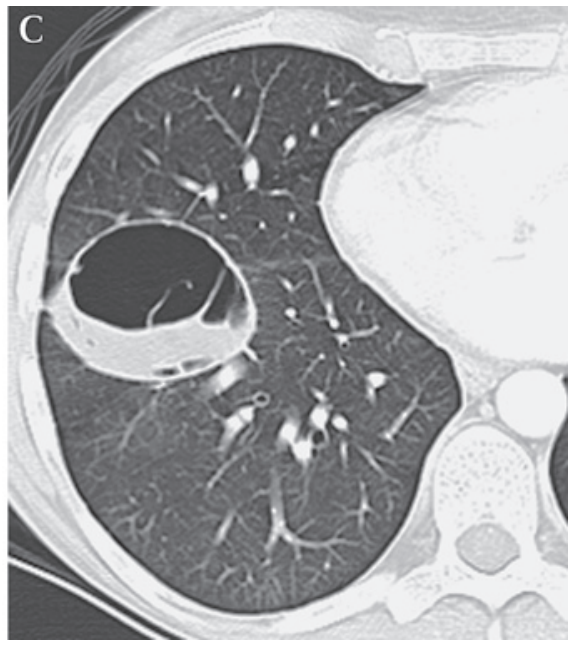

Figure 1 Pulmonary zygomycosis in a 24-year-old woman undergoing chemotherapy for recurrence of acute myelogenous leukaemia, 1 year following allogeneic stem cell transplantation, with a 2-week history of fever and dry cough. (A) Chest CT shows a focal round area of ground-glass attenuation surrounded by a ring of consolidation in the right lower lobe, consistent with the reversed halo sign. (B) Photomicrograph of the specimen from a transthoracic biopsy of the lesion shows pauciseptated hyphae with non-parallel walls and $90^{\circ}$ branching, characteristic of Zygomycetes species, which is confirmed by culture. (C) One-month follow-up CT shows cavitation of the lesion, which eventually resolved under antifungal therapy. 